\title{
International Standards of Tuberculosis Care (ISTC) - Kommentierung aus deutscher Sicht
}

\author{
International Standards of Tuberculosis Care (ISTC) - Comments from the \\ German Point of View
}

Autoren

Institute
S. Castell', T. Bauer ${ }^{1,2}$, R. Diel ${ }^{1}$, A. Hedrich ${ }^{1}$, K. Magdorf ${ }^{3}$, S. Rüsch-Gerdes ${ }^{4}$, T. Schaberg ${ }^{1,5}$, R. Loddenkemper ${ }^{1}$

Die Institutsangaben sind am Ende des Beitrags gelistet. $\begin{array}{lll}\text { eingereicht } & 12.10 .2011\end{array}$ akzeptiert nach Revision 4. 1.2012

\section{Bibliografie}

DOI http://dx.doi.org/

10.1055/s-0031-1291667

Online-Publikation: 13.3.2012

Pneumologie 2012; 66: 240-249

(c) Georg Thieme Verlag KG

Stuttgart · New York

ISSN 0934-8387

\section{Korrespondenzadresse}

\section{Dr. med. S. Castell, MSc}

Deutsches Zentralkomitee zur Bekämpfung der Tuberkulose (DZK)

Stralauer Platz 34

10243 Berlin

scastell@dzk-tuberkulose.de

\section{Zusammenfassung \\ $\nabla$}

Die „International Standards for Tuberculosis Care" (ISTC) wurden u.a. von der Weltgesundheitsorganisation (WHO) als Grundlage dafür entwickelt, dass weltweit die Betreuung von Tuberkuloseerkrankten - auch jenseits staatlicher Tuberkuloseprogramme und offizieller Gesundheitssysteme - auf Basis international abgestimmter, wenn möglich evidenzbasierter Standards erfolgt. Damit wenden sich die ISTC primär an ressourcenarme Länder mit hoher Tuberkuloseprävalenz. Hier vorgestellt wird die deutsche Übersetzung der 2009 veröffentlichten 21 Standards zu den Bereichen Diagnostik, Therapie, Koinfektion (insbesondere mit HIV) und Public Health. Die begleitenden Kommentare stellen dar, wie diese Standards in Deutschland aufgrund der hier vorhandenen medizinischen Möglichkeiten bzw. landesspezifischer Besonderheiten anzupassen sind.

Die Behandlung eines an Tuberkulose (TB) Erkrankten, im Sinne der Versorgung des einzelnen Patienten, ist das zentrale Element der Tuberkulosekontrolle, die dem Erhalt der öffentlichen Gesundheit (Public Health) dient. Die Allgemeinheit soll durch die Reduktion der Tuberkulosefälle und letztendlich Elimination der Krankheit geschützt werden [1]. Das Gesundheitssystem ist damit sowohl gegenüber dem an Tuberkulose erkrankten Individuum als auch gegenüber der Öffentlichkeit verantwortlich. Die „International Standards for Tuberculosis Care“" (ISTC) wurden als Grundlage dafür entwickelt, dass weltweit diese Ziele gerade auch jenseits staatlicher TB-Programme bestmöglich erfüllt werden können.

An dem langjährigen Prozess der Erarbeitung der Standards waren Organisationen wie die Weltgesundheitsorganisation (WHO) und "The Union“ (Internationale Union zur Bekämpfung der Tuberkulose und Lungenkrankheiten) beteiligt, das Ergebnis wurde 2006 erstmals veröffentlicht. Eine

\section{Abstract \\ $\nabla$}

The "International Standards for Tuberculosis Care" (ISTC) were developed by the World Health Organisation (WHO) and others to provide internationally agreed and, if possible, evidence-based standards for tuberculosis care including the care by private providers who are not part of national tuberculosis programmes or health-care systems. Hence, the ISTC primarily address resource-restrained countries with high tuberculosis prevalence. In this article, the German translation of the 21 standards from 2009 is presented addressing diagnostic and therapeutic standards, co-infection (especially with HIV) and publichealth issues. The accompanying comments show how these standards have to be modified for Germany due to the medical resources available here and country-specific characteristics respectively.

aktualisierte Zweitauflage erfolgte Ende 2009. Die Übersetzung der 21 Standards ins Deutsche stellen wir hiermit vor. ${ }^{1}$ Doch lassen sich diese Standards direkt auf die Situation in Deutschland übertragen? Dieser Frage geht die jeweilige Kommentierung der einzelnen Standards nach. Die Kommentierung ersetzt dabei nicht systematische Empfehlungen zur Diagnostik und Therapie der Tuberkulose.

Unabhängig von der hier vorgestellten Deutschland-spezifischen Kommentierung wurden zeitgleich auf Basis der ISTC europäische Standards (European Standards for TB Care, ESTC) von dem European Centre for Disease Prevention and Control (ECDC) und der European Respiratory Society (ERS) entwickelt [2], welche die vorliegende Kommentierung ergänzen, jedoch nicht ersetzen können.

\footnotetext{
${ }^{1}$ Das englische Orginal ist unter http://www.istcweb.org/ Home.html abrufbar.
} 


\section{Diagnostische Standards}

$\nabla$

Standard 1: Bei allen Personen mit produktivem Husten, der durch andere Ursachen nicht zu erklären ist und länger als zwei bis drei Wochen anhält, sollte eine Tuberkulose ausgeschlossen werden.

Kommentar:

Aufgrund der Tatsache, dass Tuberkulose in Deutschland im Gegensatz zu Ländern mit hoher TB-Prävalenz nur selten einem länger anhaltenden Husten als Ursache zugrunde liegt, wird der Abklärung einer Tuberkulose bei Husten keine hohe Priorität eingeräumt.

Die Leitlinie der Deutschen Gesellschaft für Pneumologie und Beatmungsmedizin (DGP) „Diagnostik und Therapie von erwachsenen Patienten mit akutem und chronischem Husten“ gibt zu dem Thema folgenden Hinweis: Erst bei länger als acht Wochen anhaltendem Husten wird die Durchführung eines Röntgenthorax in zwei Ebenen empfohlen - allerdings dann „gleich bei der ersten Vorstellung des Patienten“ [3]. Bei Husten, der weniger als acht Wochen andauert, wird eine unverzügliche weiterführende Diagnostik nur empfohlen, „wenn besondere Umstände [oder Symptome] vorliegen ${ }^{2}$, die nicht mit einem akuten banalen Infekt der Atemwege in Einklang stehen“.

Nach der Leitlinie „Husten“ der Deutschen Gesellschaft für Allgemeinmedizin und Familienmedizin (DEGAM) von 2008 sind für chronischen Husten in über $90 \%$ der Fälle in Deutschland Asthma bronchiale, bronchiale Hyperreagibilität, eine Refluxkrankheit oder ein „postnasales Drip Syndrom“ nach Ausschluss von Noxen oder der Einnahme von Husten auslösenden Medikamenten verantwortlich [4]. Nach dieser Leitlinie gilt ein Husten als chronisch, der länger als drei Wochen anhält. Eine Indikation zur Durchführung eines Röntgenthorax stellt, falls Husten auslösende Faktoren (z. B. bei Rauchern) vorliegen, die Änderung der Hustenqualität dar; in allen anderen Fällen wird die empfohlene Diagnostik aus den aus Anamnese und körperlicher Untersuchung erhobenen Verdachtsmomenten abgeleitet (z.B. Röntgenthorax, Spirometrie). Um bei der Priorisierung der diagnostischen Methoden ein erhöhtes Risiko für Tuberkulose entsprechend berücksichtigen zu können, ist die Anamnese von Risikofaktoren für Tuberkulose, wie z.B. Herkunftsland, notwendig.

Standard 2: Von allen Patienten (Erwachsene, Jugendliche und Kinder, die Sputum produzieren können), bei denen der Verdacht auf eine pulmonale Tuberkulose besteht, sollten mindestens zwei Sputen mikroskopisch in einem qualitätsgesicherten Labor untersucht werden. Wenn möglich, sollte darunter mindestens ein morgendliches Sputum sein.

\section{Kommentar:}

Für Deutschland wird die Untersuchung dreier Sputumproben empfohlen [5] - wobei morgendliches Sputum besonders geeignet ist [6], da die Sensitivität bei Verwendung von drei gegenüber zwei Sputen etwas höher liegt [7]. Über den mikroskopischen Nachweis hinaus werden - unabhängig davon, ob der mikroskopische Nachweis von säurefesten Stäbchen gelingt oder nicht Kulturen angelegt. Die ergänzende Durchführung von Nukleinsäureamplifikationstests (NAT) kann sinnvoll sein (ggf. mehrere Proben zur Verbesserung der Aussagekraft), wenn trotz Verdachts auf Lungentuberkulose kein mikroskopischer Erregernachweis

\footnotetext{
2 Die Leitlinie nennt: Hämoptoe, Thoraxschmerz, Atemnot, hohes Fieber, Aufenthalt in Ländern mit hoher Tuberkulose-Prävalenz, Kontakt zu Tuberkulosepatienten, Obdachlose, anamnestisch bekannte Malignome, Immundefizienz, HIV-Infektion, immunsuppressive Therapie, extrem starke Raucher [3].
}

vorliegt, vor allem bei Hochrisikopatienten (z.B. AIDS-Patienten, Kleinkindern) und bei schweren Verläufen (z.B. generalisierte Erkrankung) [6]. So kann eine schnelle Diagnosestellung erfolgen. In diesem Kontext wird die Sensitivität der NAT mit 80-90\% angegeben, die Spezifität mit 95 -99\% [8].

Wenn Mykobakterien nachgewiesen werden können, muss eine Bestimmung der Spezies erfolgen und eine Resistenztestung für Isoniazid (INH), Rifampicin (RMP), Ethambutol (EMB), Pyrazinamid (PZA) und Streptomycin (SM) durchgeführt werden. Wenn Resistenzen vorliegen, werden Reserveantituberkulotika geprüft [6]. Eine schnelle Resistenzprüfung auf RMP und INH, z.T. auch auf Zweitrangmedikamente (Fluorchinolone, Amikacin, Capreomycin), mittels moderner, einfach durchzuführender molekularbiologischer Techniken ist - insbesondere bei Verdacht auf das Vorliegen einer Medikamentenresistenz - zu empfehlen [8,9]. Die Sensitivität dieser Tests liegt je nach Medikament zwischen 59,0-98,1\%, die Spezifität bei 98,1-100\% [8]. Da jedoch nicht alle Medikamente (insbesondere nicht alle Zweitrangmedikamente bei Vorliegen von Resistenzen gegen Erstrangmedikamente) getestet werden können, kann auf die konventionelle Resistenztestung nicht verzichtet werden $[6,8]$.

Wenn kein Auswurf produziert werden kann oder die Sputummikroskopie negativ ausfällt, sollte ggf. ein induziertes Sputum (Inhalation von 5- bis 10\%iger Kochsalzlösung) und/oder eine Bronchoskopie vorgenommen werden; die bakteriologische Diagnostik erfolgt bei letzterer mittels Bronchialsekret oder bronchoalveolärer Lavageflüssigkeit, ggf. auch mittels Gewebeprobe (Biopsie mit zusätzlicher Histologie).

Bei Kindern, die häufig kein Sputum produzieren können, sollte vorrangig neben induziertem Sputum eine bakteriologische Untersuchung des Magennüchternsekretes oder der Magenspülflüssigkeit an drei aufeinanderfolgenden Tagen durchgeführt werden [10].

Standard 3: Von allen Patienten (Erwachsene, Jugendliche und Kinder), bei denen der Verdacht auf eine extrapulmonale Tuberkulose besteht, sollte geeignetes Material von den möglicherweise betroffenen Stellen gewonnen und mikroskopisch, kulturell sowie histopathologisch untersucht werden.

\section{Kommentar:}

Der Anteil ausschließlich extrapulmonaler Tuberkulosen lag in Deutschland 2009 an allen gemeldeten Fällen bei 19,7\% $(n=860$, nur Kinder: $23,0 \%, \mathrm{n}=32$ ). Es sind mehr Frauen betroffen als Männer (26,0\% aller Tuberkulosen bei Frauen, 15,6\% bei Männern), während bei der pulmonalen Tuberkulose das Geschlechterverhältnis umgekehrt ist [11].

Aufgrund der Tatsache, dass die extrapulmonale Tuberkulose in Deutschland noch seltener als die pulmonale Form auftritt, kann sie bei differenzialdiagnostischen Überlegungen leicht vergessen werden. Falls aus diesem Grund erst nachträglich bei zum Beispiel intraoperativ entnommenem Material, das bereits formalinfixiert ist, eine Tuberkulose-Diagnostik durchgeführt werden soll, besteht die Möglichkeit, NAT (z.B. PCR) einzusetzen [1214], wobei Sensitivität und Spezifität jedoch - im Vergleich zur Weiterverarbeitung von Originalmaterial in physiologischer Kochsalzlösung - deutlich geringer sind. Grundsätzlich sollten molekularbiologische Verfahren bei der Diagnostik der extrapulmonalen Tuberkulose in Deutschland zusätzlich zu den in Standard 3 genannten Verfahren eingesetzt werden - insbesondere wenn es sich um nicht oder nur schwer wiedergewinnbares Material (z.B. Gewebeproben, Liquor) handelt. Die Untersuchung mehrerer Proben erhöht die Aussagekraft [6]. 
Standard 4: Von allen Personen mit Tuberkulose-verdächtigen Befunden im Röntgen-Thorax sollten Sputen mikrobiologisch untersucht werden.

Kommentar:

Bezüglich der mikrobiologischen Diagnostik in Deutschland siehe Kommentar zu Standard 2.

Standard 5: Die Diagnose einer mikroskopisch negativen Lungentuberkulose sollte auf folgenden Kriterien basieren: mindestens zwei mikroskopisch unauffällige Sputumbefunde (davon mindestens ein morgendliches Sputum), Befunde im Röntgen-Thorax, die mit der Diagnose Tuberkulose zu vereinbaren sind, und fehlendes Ansprechen auf den Versuch einer Therapie mit einem Breitspektrum-Antibiotikum. Besondere Aufmerksamkeit verdient die Tatsache, dass Fluorchinolone aufgrund ihrer Wirksamkeit gegen den Mycobacterium tuberculosis-Komplex (M. tb.-Komplex) in diesem Zusammenhang vermieden werden sollen, da sie bei an Tuberkulose Erkrankten zu einer vorübergehenden Verbesserung führen können. Bei Patienten mit Verdacht auf eine pulmonale Tuberkulose mit fehlendem mikroskopischem Nachweis sollten Kulturen der Sputen angelegt werden. Bei Menschen, die schwer erkrankt sind oder bei welchen eine HIV-Infektion bekannt ist bzw. ein diesbezüglicher Verdacht besteht, sollte die Diagnostik beschleunigt werden. Bei ausgeprägten klinischen Anhaltspunkten für eine Tuberkulose sollte eine antituberkulöse Behandlung eingeleitet werden.

\section{Kommentar:}

In Deutschland ist der Versuch einer Breitspektrum-Antibiose bei Verdacht auf Tuberkulose im Sinne eines ungezielten Behandlungsversuches nicht üblich und aufgrund der Verzögerung der Diagnostik nicht Teil des diagnostischen Ablaufs [3]. Bei klinischem und/oder auf der Bildgebung (Röntgen und Computertomographie, s.u.) beruhendem Verdacht auf eine (mikroskopisch und in der PCR negative) Tuberkulose ohne anamnestische Hinweise auf das Vorliegen resistenter Erreger sollte nach Durchführung einer Bronchoskopie (s. Kommentar zu Standard 2) mit der Therapie begonnen werden, die im weiteren Verlauf klinisch, bakteriologisch und röntgenologisch reevaluiert und ggf. geändert werden muss.

Gerade für Patienten, deren Sputen mikroskopisch negativ sind oder die kein Sputum produzieren können, kann in einem Land wie Deutschland (niedrige Tuberkulose-Inzidenz, niedrige HIVPrävalenz, ausreichende medizinische Ressourcen) der Einsatz der hochauflösenden Computertomographie (HRCT) in Kombination mit einem Interferon-gamma Release-Assay (IGRA) einen großen diagnostischen Nutzen haben. Es konnte gezeigt werden, dass die Kombination dieser beiden diagnostischen Verfahren einen positiven prädiktiven Wert von 96\% für eine behandlungsbedürftige Lungentuberkulose (Läsionen im HRCT, die einen TBVerdacht nahelegen, und positives IGRA-Testresultat) bzw. einen negativen prädiktiven Wert von $92 \%$ (keine auffälligen Läsionen im HRCT und negatives IGRA-Testresultat) aufweist [15]. Allerdings wird bis auf definierte Sonderfälle die Diagnostik mittels IGRA im ambulanten Bereich nicht durch die gesetzliche Krankenversicherung (GKV) übernommen [16].

Standard 6: Bei allen Kindern, bei welchen der Verdacht auf eine intrathorakale (d.h. Lunge, Pleura oder mediastinale, hiläre Lymphknoten betreffende) TB besteht, sollte dies bakteriologisch (Mikroskopie und Kultur) bestätigt werden (Untersuchung von Auswurf, Magensaft oder induziertem Sputum). Bei negativen Resultaten dieser Untersuchungen sollte sich die Diagnose Tuberkulose auf Auffälligkeiten im Röntgen-Thorax, die zur Erkrankung
Tuberkulose passen, auf eine positive Kontaktanamnese (Kontakt mit einem infektiösen Indexpatienten), den Nachweis einer tuberkulösen Infektion (positiver Tuberkulinhauttest oder Interferongamma Release-Assay) und klinische Befunde, die auf eine TB hindeuten, stützen. Bei Kindern mit Verdacht auf extrapulmonale TB sollte geeignetes Material zur mikroskopischen, kulturellen und histopathologischen Untersuchung gewonnen werden.

\section{Kommentar:}

Gewebeproben, die vor Therapiebeginn (zusätzlich Histopathologie: Formalin) zur mikrobiologischen Diagnostik gewonnen werden, sind in physiologischer Kochsalzlösung zu transportieren (z.B. periphere Lymphknoten).

Für Deutschland ist außerdem zu ergänzen, dass bei Verdacht auf eine mediastinale Lymphknoten-Tuberkulose ggf. eine endobronchiale Ultraschalluntersuchung (EBUS) mit Punktion der Lymphknoten vorgenommen werden kann. Bei Pleuritis tuberculosa kann eine Pleurastanzbiopsie/Thorakoskopie, ggf. mit Lösung der Fibrinsegel, vorgenommen werden. Letztere wird neben der besseren Diagnostik (gezielte Gewebsproben, häufigeres Gelingen des kulturellen Nachweises) auch therapeutisch durch anschließende Pleuradrainagen, die einer Pleuraverklebung vorbeugen können, eingesetzt [17]. Bei Vorliegen eines Pleuraergusses sollte zunächst eine diagnostische Pleurapunktion erfolgen [18]. Darüber hinaus bietet die moderne Bildgebung (hochauflösendes CT) in komplizierten Fällen eine weitere diagnostische Möglichkeit (s.o.) [19]. Ein grundsätzlich sparsamer Einsatz von Röntgenstrahlen gerade bei Kindern ist dabei selbstverständlich. Bezüglich der Diagnostik extrapulmonaler Tuberkulosen siehe Kommentar zu Standard 3. Bezüglich des exakten diagnostischen Vorgehens bei Kindern sei zusätzlich auf pädiatrische Spezialliteratur verwiesen [10].

\section{Standards für die Behandlung}

\section{$\nabla$}

Standard 7: Jeder Arzt, der einen Tuberkulosepatienten behandelt, übernimmt auf dem Gebiet der öffentlichen Gesundheit (Public Health) die wichtige Verantwortung, eine fortlaufende Übertragung der Infektion und die Entwicklung von Medikamentenresistenzen zu verhindern. Hierfür muss er/sie nicht nur die geeignete Medikamentenkombination verschreiben, sondern auch den zuständigen Öffentlichen Gesundheitsdienst und - falls notwendig - andere Behörden hinzuziehen, um die Therapieadhärenz des Patienten einzuschätzen und ggf. mangelhafter Adhärenz zu begegnen.

\section{Kommentar:}

In Deutschland besteht für Ärzte nach dem Infektionsschutzgesetz (IfSG) §6 Abs.1 eine namentliche Meldepflicht, wenn eine behandlungsbedürftige Tuberkulose (oder der Tod daran) festgestellt wird [20]. Dies ist spätestens bei Einleitung der antituberkulösen Therapie der Fall. Die Zusammenarbeit der behandelnden Ärzte mit dem Öffentlichen Gesundheitsdienst (ÖGD, Gesundheitsamt) ist also verpflichtend und geht über die allgemein gehaltene Empfehlung von Standard 7 der ISTC hinaus. Darüber hinaus besteht für mikrobiologische Labore (IfSG $§ 7$ Abs. 1) und Pathologen (IfSG $\S 8$ Abs.3) Meldepflicht. Auch die Verweigerung oder der Abbruch der Behandlung sind meldepflichtig (IfSG §6 Abs.2). Die Einschätzung der Therapieadhärenz erfolgt primär durch den behandelnden Arzt. Das Gesundheitsamt ist zusätzlich für die Sicherstellung der Behandlung zuständig, außerdem für die Infektionskontrolle (Umgebungsuntersuchung und Verhütung weiterer Infektionen durch den gemeldeten $\mathrm{Pa}$ tienten) $[5,21]$. 
Standard 8: Alle Patienten (auch HIV-infizierte) ohne vorhergehende antituberkulöse Therapie sollen eine international anerkannte Kombinationstherapie aus Erstrangmedikamenten mit bekannter Bioverfügbarkeit erhalten. Initial sollten Isoniazid (INH), Rifampicin (RMP), Pyrazinamid (PZA) und Ethambutol (EMB) über zwei Monate verabreicht werden, in der sich anschließenden Kontinuitätsphase Isoniazid und Rifampicin über vier Monate. Die Dosierungen sollten internationalen Empfehlungen entsprechen. Fixe Medikamentenkombinationen von zwei (Isoniazid und Rifampicin), drei (Isoniazid, Rifampicin, Pyrazinamid) oder vier (Isoniazid, Rifampicin, Pyrazinamid, Ethambutol) Medikamenten werden sehr empfohlen.

\section{Kommentar:}

Die empfohlene Therapie entspricht der in Deutschland verwendeten antituberkulösen Standardtherapie für Patienten, bei welchen kein Verdacht auf das Vorliegen einer resistenten Tuberkulose besteht [22]. Allerdings werden fixe Medikamentenkombinationen nicht empfohlen, individuelle Dosierungen sind damit nicht möglich. Für das Vorgehen bei Hinweisen auf das Vorliegen von Medikamentenresistenzen (oder -unverträglichkeit), bei relevanten Komorbiditäten und möglichen Medikamenteninteraktionen oder auch bei Kindern wird auf Spezialliteratur verwiesen [22].

Standard 9: Um die Therapieadhärenz einzuschätzen und zu unterstützen, sollte ein patientenzentriertes Vorgehen bei Verordnung und Gabe der Medikamente entwickelt werden, das den Patienten in den Mittelpunkt stellt, auf seine Bedürfnisse zugeschnitten ist und auf gegenseitigem Respekt zwischen dem Patienten und dem Behandler beruht. Supervision und Unterstützung sollten individuell erfolgen und sämtliche empfohlene Interventionen und vorhandene Unterstützungsmöglichkeiten inklusive Patientenberatung und -schulung sind einzubeziehen. Ein zentrales Element der patientenbezogenen Strategie sind Maßnahmen zur Einschätzung und Förderung der Behandlungsadhärenz sowie zur Intervention bei mangelnder Adhärenz. Diese Maßnahmen sollten auf die Lebenslage des jeweiligen Patienten zugeschnitten und für Patient und Behandler akzeptabel sein.

Es kann sich hierbei um eine überwachte Medikamenteneinnahme („directly observed treatment“, DOT) handeln sowie um die Identifizierung und Anleitung einer Person, welche die Therapie der Tuberkulose (und wenn erforderlich der HIV-Koinfektion) unterstützt, die vom Patienten und dem Gesundheitswesen akzeptiert wird und beiden gegenüber rechenschaftspflichtig ist. Adäquate Anreize und Hilfen, inklusive finanzieller Unterstützung, können zusätzlich dazu dienen, die Therapieadhärenz zu verstärken.

\section{Kommentar:}

Die Verantwortung des ÖGD in Bezug auf den Infektionsschutz lässt sich aus $\S \S 16$ und 28 IfSG ableiten [20]. Umgesetzt wird der Schutz der Allgemeinheit u. a. durch die Sicherstellung einer erfolgreich durchgeführten Therapie. Im Rahmen der stationären Therapie wird die überwachte Einnahme der antituberkulösen Medikamente empfohlen [21,22]. Bei Entlassung aus dem Krankenhaus ist nach §9 (1) Nr. 12 IfSG dem Gesundheitsamt der Aufenthaltsort des Patienten zu melden. Dieses ist dann - im Sinne einer Koordinationsstelle - für die Sicherstellung der Kontinuität der ärztlichen Behandlung, die Gewährleistung der Fortführung der medikamentösen Therapie ${ }^{3}$ und ggf. für die überwachte Medikamenteneinnahme, sollte diese nicht zuverlässig gewährleistet sein, zuständig. Eine Behandlung im Sinne der „direkt überwachten Therapie“ („directly observed treatment“, DOT) wird in Deutschland - nach Ermessen - nur bei besonderen Risikogrup- pen wie Suchtkranken, psychisch Kranken, senilen Menschen, bei Wiederaufnahme der Therapie nach Behandlungsabbruch oder im Justizvollzug durchgeführt, in Sonderfällen auch bei Kindern $[21,23]$. Ort der ambulanten überwachten Medikamenteneinnahme kann das Gesundheitsamt sein, jedoch auch die Praxis des behandelnden Arztes, eine Sozialstation oder ein Obdachlosenheim; außerdem kann die Supervision von einem ambulanten Pflegedienst übernommen werden [21]. Eine adäquate Finanzierung der direkt überwachten Therapie im ambulanten Bereich ist gegenwärtig nur bei pflegebedürftigen Personen unter bestimmten Bedingungen gemäß Richtlinie über die Verordnung von häuslicher Krankenpflege in der vertragsärztlichen Versorgung nach §92 Abs. 1 (Satz 2, Nr.6) und Abs. 7 SGB V gewährleistet [24].

Die persönlichen Kontakte und der Aufbau einer tragfähigen Beziehung zwischen Ärzten oder Mitarbeitern des Gesundheitsamtes und Patient sind dabei von zentraler Bedeutung. Auch das persönliche Gespräch zwischen behandelndem Arzt und Mitarbeitern des ÖGD tragen wesentlich zu einer koordinierten und erfolgreichen Behandlung bei. Eine direkt überwachte Therapie ist dabei keine Anordnung auf gesetzlicher Basis, sondern eine Maßnahme, die zwischen den beteiligten Parteien im Sinne der freiwilligen Sicherung des Behandlungserfolges vereinbart wird. Es soll sich also nicht um eine repressive Maßnahme gegenüber dem Patienten handeln, sondern um ein Angebot und eine Unterstützung des Patienten.

Weitere Möglichkeiten zur Unterstützung von Tuberkulosepatienten sind beispielsweise Hilfen zur Regelung behördlicher Angelegenheiten. Über $\S 30$ (5) SGB XII kann Mehrbedarf für Arbeitlosengeld II-Empfänger für die Dauer der Therapie beantragt werden (kostenaufwändige Ernährung) [25]. Allerdings sind derartige Maßnahmen nicht Teil eines strukturierten oder gar gesetzlich geregelten Belohnungssystems (sogenannte „incentives“ oder „enabler“), wie es in manchen Projekten oder Ländern zur Anwendung kommt (zum Beispiel finanzielle Unterstützung, wenn die Behandlung erfolgreich beendet wurde).

Falls Patienten die Behandlung abbrechen, wird bei nicht-infektiösen Tuberkulosen eine Überwachung des Infektionsstatus und der Krankheitsprogredienz mittels regelmäßiger Röntgenthorax- und Sputumuntersuchung (§29 IfSG) durchgeführt ${ }^{4}$. Bei infektiösen Tuberkulosen kann nach §30 IfSG eine sogenannte zwangsweise Absonderung nach richterlichem Beschluss angeordnet werden. Entsprechende Einrichtungen befinden sich in Parsberg und in Bad Lippspringe.

Standard 10: Das Ansprechen auf die Therapie sollte bei Patienten mit pulmonaler Tuberkulose mittels zweier weiterer mikroskopisch untersuchter Sputen am Ende der Initialphase, also zwei Monate nach Behandlungsbeginn, kontrolliert werden. Wenn sich im Sputum zu diesem Zeitpunkt säurefeste Stäbchen mikroskopisch nachweisen lassen, sollte eine weitere Untersuchung einen Monat später stattfinden. Ist diese mikroskopisch wieder positiv, sollten Kulturen angelegt und eine Medikamentenresistenzprüfung durch-

\footnotetext{
${ }^{3}$ Nach §19 IfSG ist eine (vorläufige) Kostenübernahme der Therapie (öffentliche Mittel) in Einzelfällen (Personen, deren Lebensumstände eine erhöhte Ansteckungsgefahr für andere mit sich bringen) möglich, wenn der Patient (gegenwärtig) nicht krankenversichert ist und die Kosten der Behandlung nicht selbst tragen kann.

${ }^{4}$ Allerdings ist zu berücksichtigen, dass unzureichend behandelte Patienten, die zum Zeitpunkt der Therapieabbruchs nicht (mehr) infektiös sind, im weiteren Verlauf erneut progredieren und unter Umständen (wieder) ansteckungsfähig werden können.
} 
geführt werden. Bei Patienten mit extrapulmonaler Tuberkulose und bei Kindern kann das Ansprechen auf die Therapie am besten klinisch überprüft werden.

\section{Kommentar:}

Kulturen sollten in Deutschland - bei Tuberkulosen mit Erregernachweis - bereits von dem vor Therapiebeginn gewonnenen Material angelegt werden. Damit kann und sollte eine Resistenztestung auf Erstrangmedikamente (s. o.) grundsätzlich bei jedem Patienten mit Nachweis von M. tuberculosis-Komplex erfolgen. Zur Therapieverlaufskontrolle werden mikroskopische Kontrollen alle zwei Wochen empfohlen, die erneute Anlage einer Kultur soll vier, acht und zwölf Wochen nach Therapiebeginn erfolgen [22]. Der hier vorgestellte Standard 10 empfiehlt eine Resistenztestung bei mikroskopisch positiv bleibendem Sputum nach drei Monaten. Gegenwärtig wird für Deutschland empfohlen, bei kulturellem Nachweis von Mycobacterium tuberculosis noch nach acht Wochen Behandlung eine erneute Resistenztestung (genound phänotypisch) durchzuführen [22].

Zum Nachweis des Therapieerfolgs sollte - bei Tuberkulose mit Erregernachweis - eine Dokumentation der kulturellen Konversion (jeweils am Ende der Initial- und der Kontinuitätsphase ${ }^{5}$ ) versucht werden $[22,26]$. Dies kann sich allerdings, wenn sich unter Therapie der Auswurf reduziert, schwierig gestalten ${ }^{6}$. Zusätzlich zu der genannten bakteriologischen Therapieverlaufskontrolle sollten bei pulmonalen Tuberkulosen Röntgenverlaufskontrollen erfolgen. Bezüglich des genauen Vorgehens - auch hinsichtlich Laborkontrollen - wird auf die Spezialliteratur verwiesen [22].

Im Fall von extrapulmonalen Tuberkulosen erfolgt die Einschätzung des Therapieerfolges, wie im Standard 10 empfohlen, klinisch (Ausnahme: bakteriologische Kontrollen bei Urogenital-Tuberkulose) [22]. Zusätzlich sollten bildgebende Verfahren zum Einsatz kommen.

Standard 11: Die Wahrscheinlichkeit für das Vorhandensein einer resistenten Tuberkulose sollte bei allen Patienten auf Basis einer Vorbehandlung wegen Tuberkulose, eines möglichen Kontaktes mit einem Tuberkulose-Fall mit nachgewiesen resistenten Bakterien sowie der regionalen Prävalenz von Resistenzen eingeschätzt werden. Eine Resistenztestung sollte bei allen vorbehandelten Patienten vor dem Beginn der Therapie durchgeführt werden. Patienten, die nach drei Monaten Behandlung weiterhin sputumpositiv sind, und Patienten, bei welchen die Behandlung versagt ${ }^{7}$, welche die Behandlung abbrechen ${ }^{8}$, oder bei welchen nach einem oder mehreren Behandlungszyklen ein Krankheitsrezidiv auftritt, sollten stets auf Medikamentenresistenz untersucht werden. Bei Patienten, bei welchen das Auftreten einer medikamentenresistenten TB wahrscheinlich ist ( $\bullet$ Tab.1), sollten umgehend Kulturen angelegt und eine

\footnotetext{
${ }^{5}$ Um einen Patienten als geheilt klassifizieren zu können, wird der Nachweis einer negativen Kultur im letzten Behandlungsmonat und mindestens zu einem früheren Zeitpunkt gefordert [27].

6 Bei fehlendem Nachweis der Sputumkonversion wird der Therapieerfolg nach internationalen Richtlinien $[27,28]$, wenn die vorgesehene Behandlung vollständig durchgeführt wurde, als solcher klassifiziert. Eine klinische und röntgenologische Besserung sollte allerdings zusätzlich dokumentiert sein.

7 Anmerkung: Definition des Therapieversagens nach [28]: Mikroskopieoder Kulturpositivität nach fünf oder mehr Monaten Therapie und bei Patienten, die eine MDR-TB unter Therapie entwickelt haben.

${ }^{8}$ Anmerkung: Definition nach [28]: Therapieunterbrechung für mind. zwei Monate.
}

Resistenztestung (zumindest auf Isoniazid und Rifampicin) ${ }^{9}$ durchgefüht werden. Die Beratung und Schulung des Patienten sollte in einem solchen Fall sofort beginnen, um die Möglichkeit einer Übertragung zu minimieren. Der Situation angemessene Maßnahmen der Infektionskontrolle sollten angewendet werden.

\section{Kommentar:}

Da die Prävalenz der (resistenten) Tuberkulose in Deutschland vom Herkunftsland des Patienten bzw. der Zusammensetzung von Nationalitäten in der Bezugsgemeinschaft des Patienten abhängt, sind diese Aspekte in der Anamnese zu erfragen und entsprechend $\mathrm{zu}$ berücksichtigen. ${ }^{10}$ Zur Risikoeinschätzung einzelner Länder sei auf Publikationen der WHO zur globalen Resistenzlage verwiesen (z.B. [29] und http://www.who.int/tb/country/data/profiles/en/index.html: Möglichkeit, die MDR-TB-Anteile länderbezogen abzufragen).

Bei Vorbehandlungen ist die genaue Medikamentenanamnese wichtig, bei im Ausland durchgeführten Behandlungen jedoch zum Teil schwer zu verifizieren. Die in $\bullet$ Tab. 1 genannten Risikofaktoren sollten auch in Deutschland beachtet werden. Allerdings ist i.d.R. kein gesteigertes Risiko für eine resistente Tuberkulose bei Obdachlosen, Ärzten/Pflegepersonal/Laborpersonal bekannt.

Wie bereits erwähnt, sollte bei jeder Erkrankung an Tuberkulose mit Erregernachweis eine (Schnell-)Resistenztestung (Erstrangmedikamente, bei Nachweis von Resistenzen auch von Zweitrangmedikamenten) aus vor Therapiebeginn gewonnenem Material erfolgen.

Es gelten die für Tuberkulose spezifischen Maßnahmen zur Infektionsverhütung [30].

Standard 12: Patienten mit Verdacht auf oder mit nachgewiesener resistenter Tuberkulose (insbesondere $M D R / X D R^{11}$ ) sollten mit einer speziellen Medikamentenkombination behandelt werden, die antituberkulöse Zweitrangmedikamente enthält. Die gewählte Medikamentenkombination kann standardisiert sein oder auf vermuteten oder nachgewiesenen Resistenzmustern basieren. Es sollten mindestens vier Medikamente, für welche die Organismen nachgewiesen oder vermutlich sensibel sind, inklusive einem injizierbaren Medikament, verwendet werden und die Behandlung sollte mind. 18-24 Monate über die Kulturkonversion hinaus erfolgen. Patientenzentrierte Maßnahmen, inklusive überwachter Therapie, sind zur Gewährleistung der Therapieadhärenz erforderlich. Die behandelnden Ärzte sollten sich mit einem in der Behandlung von MDR- und XDR-TB erfahrenen Experten beraten.

\section{Kommentar:}

Gerade im Rahmen der Therapie der (mehrfach) resistenten Tuberkulose sollte ein individualisiertes Vorgehen unter Beachtung der nachgewiesenen Resistenzen angestrebt werden. Dabei ist bei vorbehandelten Patienten - neben der Beachtung der Laborresultate unbedingt die Medikamentenanamnese der Vorbehandlung - soweit möglich - zu berücksichtigen, um ggf. Diskrepanzen zwischen Befunden und Anamnese nachgehen zu können.

\footnotetext{
${ }^{9}$ MDR-TB (“multidrug resistant”): Resistenz gegen mindestens INH und RMP [22].

${ }^{10}$ Beispielsweise liegt der prozentuale Anteil der MDR-Fälle (Definition siehe unten) an allen neu aufgetretenen Fällen für in Deutschland geborene Personen bei $0,8 \%$, für in Ländern der ehemaligen Sowjetunion geborene bei 13,2\% und bei aus „weiteren Ländern“ stammenden bei 1,2\% [11].

11 XDR-TB (“extensively drug resistant”): MDR-TB und zusätzlich Resistenz gegen mindestens ein Fluorchinolon und eins der drei injizierbaren Zweitrangmedikamente Amikacin, Capreomycin und Kanamycin [22].
} 


\begin{tabular}{|c|c|}
\hline Risikofaktoren für Resistenzen & Kommentare (im Orginaltext der ISTC) \\
\hline Versagen eines erneuten Behandlungsregimes ${ }^{1}$ & $\begin{array}{l}\text { Patienten, die am Ende eines erneuten Therapieregimes immer noch } \\
\text { sputumpositiv sind, haben möglicherweise den höchsten MDR-TB-Anteil } \\
\text { von allen Gruppen. Dieser liegt oft bei mehr als } 80 \% \text {. }\end{array}$ \\
\hline $\begin{array}{l}\text { Enger Kontakt zu einem Patienten mit } \\
\text { bekannter resistenter Tuberkulose }\end{array}$ & $\begin{array}{l}\text { Die meisten Studien haben gezeigt, dass eine Tuberkulose, die bei engen } \\
\text { Kontaktpersonen von MDR-TB-Patienten auftritt, mit hoher Wahrschein- } \\
\text { lichkeit auch eine MDR-TB ist. }\end{array}$ \\
\hline Versagen des initialen Behandlungsregimes & $\begin{array}{l}\text { Patienten, die während einer Behandlung nicht sputumnegativ werden, } \\
\text { sind wahrscheinlich an medikamentenresistenten Bakterien erkrankt. } \\
\text { Diese Wahrscheinlichkeit hängt jedoch von diversen Faktoren ab, unter } \\
\text { anderem ob Rifampicin in der Kontinuitätsphase eingesetzt wurde und ob } \\
\text { eine überwachte Therapie während der gesamten Behandlung erfolgte. } \\
\text { Aus diesem Grunde ist eine exakte Anamnese bezüglich der verwendeten } \\
\text { Medikamente wichtig. Das gilt besonders für Patienten, die von privaten } \\
\text { Ärzten² behandelt worden waren - oft ohne Einsatz von Standard- } \\
\text { therapien. }\end{array}$ \\
\hline $\begin{array}{l}\text { Rezidiv nach anscheinend erfolgreicher } \\
\text { Behandlung }\end{array}$ & $\begin{array}{l}\text { Innerhalb klinischer Studien lassen sich bei den meisten Patienten, die } \\
\text { erneut erkranken, voll sensible Bakterien nachweisen. Außerhalb von } \\
\text { Studien ist ein Rezidiv, insbesondere ein frühes, jedoch ein Hinweis auf ein } \\
\text { nicht erkanntes Therapieversagen und daher mit einer höheren Wahr- } \\
\text { scheinlichkeit für Resistenzen verbunden. }\end{array}$ \\
\hline $\begin{array}{l}\text { Wiederbeginn der Medikamenteneinnahme } \\
\text { nach Therapieabbruch ohne kürzliches } \\
\text { Therapieversagen }\end{array}$ & $\begin{array}{l}\text { Die Wahrscheinlichkeit für eine MDR-TB variiert in dieser Gruppe sehr und } \\
\text { ist zum Teil abhängig von der Dauer der erfolgten Behandlung sowie von } \\
\text { dem Grad der Adhärenz vor dem Therapieabbruch. }\end{array}$ \\
\hline $\begin{array}{l}\text { Exposition innerhalb von Institutionen, } \\
\text { die Ausbrüche von resistenter Tuberkulose } \\
\text { oder eine hohe Prävalenz resistenter } \\
\text { Tuberkulose haben }\end{array}$ & $\begin{array}{l}\text { Patienten, die regelmäßig in Obdachlosenheimen übernachten, in vielen } \\
\text { Ländern Gefangene und Personen, die im Gesundheitssystem (Ambulan- } \\
\text { zen, Labore, Krankenhäuser) arbeiten, können einen hohen Anteil von } \\
\text { resistenter Tuberkulose aufweisen. }\end{array}$ \\
\hline $\begin{array}{l}\text { Wohnhaft in Regionen mit hoher Prävalenz } \\
\text { resistenter Tuberkulosen }\end{array}$ & $\begin{array}{l}\text { Die Anteile an resistenter Tuberkulose sind in vielen Regionen der Welt hoch } \\
\text { genug, um die routinemäßige Resistenztestung zu rechtfertigen. }\end{array}$ \\
\hline
\end{tabular}

Die Länge der Behandlung einer MDR-TB sollte sich auch in Deutschland an den von der WHO herausgegebenen Leitlinien orientieren, die von den Angaben des Standards 12 abweichen [31]. In dem WHO „2011 update“ wird eine Dauer von mindestens 20 Monaten mit einer Initialphase (Verwendung eines injizierbaren Zweitrangmedikaments) von 8 Monaten empfohlen. Bezüglich der Medikamentenkombination und des genauen therapeutischen Vorgehens sei auf die Literatur verwiesen [22,31]. Die Behandlung von XDR-TB kann seit Februar 2012 durch Bedaquilin ${ }^{\circledR}$ (Tibotec) i.S. eines „compassionate use“ ergänzt werden (www.bfarm.de).

Die langwierige Therapie einer mehrfach resistenten Tuberkulose wird z.T. schlecht vertragen. Aus diesem Grund ist die verantwortliche Mitarbeit des Patienten gefordert, die vom medizinischen Personal und den Mitarbeitern des ÖGD gefördert werden muss (siehe Kommentar zu Standard 9).

In Deutschland seltener angewandt, aber gerade bei schwer zu behandelnden Tuberkulosen als Therapiemöglichkeit mit in Betracht zu ziehen, ist unter Berücksichtigung funktioneller Aspekte die chirurgische Intervention [22,32].

Die Behandlung von MDR- und XDR-Tuberkulosen sollte nur in damit erfahrenen Zentren vorgenommen werden. Das gesamte Management von Tuberkulosen, die mit Zweitrangmedikamenten behandelt werden müssen, sollte durch ein Team von mehreren Experten erfolgen, um Fehler zu minimieren und angesichts niedriger Prävalenzen Expertise zu maximieren.

Standard 13: Eine schriftliche Dokumentation aller verabreichten Medikamente, des bakteriologischen Ansprechens und der Nebenwirkungen sollte für alle Patienten regelmäßig erfolgen. werden können.
Tab. 1 Risikofaktoren für Resistenzen (ISTC). zur Therapie (Therapieregime, Veränderungen im Regime, bakteriologische Befunde einschließlich Resistenzen u.a.) eingetragen

\section{Standards, die sich mit HIV-Infektion und weiteren Komorbiditäten befassen \\ $\nabla$}

Standard 14: Die Durchführung eines HIV-Tests und einer entsprechenden Beratung wird für alle Patienten mit nachgewiesener oder vermuteter Tuberkulose empfohlen. Dies ist ein besonders wichtiger Bestandteil des Routinemanagements aller TB-Patienten in Re- 
gionen mit hoher HIV-Prävalenz in der Allgemeinbevölkerung, bei Patienten mit Symptomen oder Zeichen einer mit HIV assoziierten Erkrankung und bei Hinweisen auf ein hohes Risiko für eine HIVExposition in der Anamnese. Aufgrund des engen Zusammenhangs zwischen Tuberkulose und HIV-Infektion werden in Regionen mit hoher HIV-Prävalenz integrierte Ansätze zur Prävention und Behandlung beider Infektionen empfohlen.

Kommentar:

Das Angebot der Durchführung eines HIV-Testes sollte in Deutschland, wie in Standard 14 formuliert, bei Tuberkulose generell erfolgen [22]. Bei Hinweisen in der Anamnese auf Risikofaktoren für virale Hepatitis wie z. B. intravenösen Drogenmissbrauch sollte zusätzlich eine Hepatitis-Serologie erfolgen, da die Tuberkulosebehandlung ein hepatotoxisches Potenzial hat und je nach Befundkonstellation diese Patienten gezielt überwacht werden müssen [22].

Standard 15: Alle Patienten mit Tuberkulose und HIV-Infektion sollten bezüglich der Indikation für eine antiretrovirale Therapie während der Tuberkulosebehandlung evaluiert werden. Bei Vorliegen einer solchen Indikation sollten die Patienten entsprechend Zugang $z u$ antiretroviralen Medikamenten erhalten, ohne dabei den Beginn der antituberkulösen Therapie zu verzögern. Patienten mit Tuberkulose und HIV-Infektion sollten zusätzlich Cotrimoxazol als Prophylaxe für andere Infektionen erhalten.

\section{Kommentar:}

Bei Erkrankung eines HIV-Patienten an Tuberkulose liegt das AIDS-Krankheitsstadium C (nach der Einteilung durch die Centers for Disease Control and Prevention (CDC), USA) vor. In diesem Fall soll unabhängig von der CD4-Zellzahl mit der antiretroviralen Therapie (ART) begonnen werden, falls diese nicht bereits verabreicht wird [35]. Die aktuellen Richtlinien der WHO, die primär ressourcenschwache Länder als Zielgruppe haben, empfehlen bei bislang unbehandelten koinfizierten Patienten zuerst die Aufnahme der antituberkulösen Behandlung und im zweiten Schritt (sobald wie möglich innerhalb von acht Wochen) ergänzend den Beginn von ART [28]. Der Grund dafür ist, Nebenwirkungen entweder auf die Tuberkulosetherapie oder ART zurückführen zu können, Medikamentenwechselwirkungen zu Beginn zu vermeiden und die Wahrscheinlichkeit für das Auftreten eines Immunrekonstitutionssyndroms (IRIS) zu reduzieren (s.u.). Die Ausführungen der CDC zu dem Thema der Koordinierung von TB-Therapie und ART differenzieren in Abhängigkeit von der CD4-Zellzahl: Unter 100 Zellen/Mikroliter wird die ergänzende Aufnahme von ART frühestens zwei Wochen nach Beginn der TB-Therapie empfohlen, bei 100-200 Zellen/Mikroliter nach Vollendung der Initialphase der TB-Therapie, bei 200-350 Zellen/Mikroliter im Verlauf der Kontinuitätsphase und bei mehr als 350 Zellen/Mikroliter nach Beendigung der antituberkulösen Behandlung [36]. Aktuelle Studien weisen darauf hin, dass bei ART-naiven koinfizierten Patienten mit fortgeschrittener Immunsuppression der Beginn der antiretroviralen Therapie schon möglichst früh (innerhalb von zwei Wochen) während der Tuberkulosebehandlung vorteilhaft sein kann [37]. Da in Deutschland bei schätzungsweise 1000 Personen pro Jahr die HIV-Erstdiagnose im Stadium von AIDS oder mit einer CD4-Zellzahl von unter 200/Mikroliter gestellt wird [38], kann diese Empfehlung für eine kleine Patientengruppe von Relevanz sein.

Zu beachten ist, dass nach Beginn der ART durch die zunehmende Immunkompetenz bei persistierender Erregerlast ein IRIS auftreten kann. Dieses resultiert aus einer schnellen Wiederherstellung der pathogenspezifischen Immunantwort auf opportunistische
Infektionen durch die antiretrovirale Therapie bei $25-60 \%$ der Fälle [39]. Allerdings verläuft die Symptomatik bei pulmonaler Tuberkulose meist mild und kann in der Regel ohne medizinische Intervention bewältigt werden [28,37], während eine neurologische Beteiligung - in ca. $10 \%$ der IRIS-Patienten vorhanden - mit schwereren Verläufen verbunden ist [40].

Betroffen von einem IRIS sind insbesondere Patienten mit schwerster Immunschwäche (CD4-Zellen unter 100/Mikroliter), einem raschen Anstieg der T-Zellen und disseminierter Tuberkulose [39]. Als weitere Risikofaktoren für ein IRIS werden extrapulmonale Tuberkulosen sowie ein geringer Abstand zwischen Beginn der TB-Therapie und der ART genannt [37,41]. Abhängig vom klinischen Bild sind zusätzlich Kortikosteroide einzusetzen [22].

Es wird empfohlen, im Falle einer Koinfektion mit HIV die antituberkulöse Kontinuitätsphase (Zweifachtherapie) von vier auf sieben Monate zu verlängern [22]. Die Gabe von Cotrimoxazol ist ab einer T-Helferzellzahl von unter 200/Mikroliter indiziert und von einer Erkankung an Tuberkulose unabhängig [42].

Standard 16: HIV-Infizierte sollen nach sorgfältigem Ausschluss einer aktiven Tuberkulose bei vermuteter latenter Tuberkuloseinfektion mit Isoniazid über 6-9 Monate behandelt werden.

\section{Kommentar:}

Bei HIV-positiven Menschen mit einer CD4-Anzahl von > 350 Zellen/Mikroliter ist davon auszugehen, dass die klinische Manifestation der Tuberkulose derjenigen von HIV-negativen Patienten gleicht [36]. Zu beachten ist bei der Einschätzung der Situation, dass grundsätzlich bei HIV-positiven Menschen extrapulmonale Tuberkulosen häufiger sind. Darüber hinaus bestehen beim Fortschreiten der Immunschwäche Schwierigkeiten in der Diagnostik der Tuberkulose: Die röntgenologischen, bakteriologischen und histopathologischen Untersuchungen können trotz aktiver Tuberkulose atypisch oder unauffällig sein [22,39]. Falsch negative Testresultate sind aufgrund der Immunsuppression sowohl beim Tuberkulinhauttest (THT) als auch beim IGRA möglich [36]. Bei Letzterem treten zusätzlich in ca. 4-6\% der Fälle auch unklare Resultate auf [43].

Bei der Erstdiagnose einer HIV-Infektion sollte auf latente tuberkulöse Infektion (LTBI) bzw. manifeste Erkrankung untersucht werden. Dies sollte im weiteren Verlauf laut Empfehlungen der CDC jährlich wiederholt werden, wenn ein erhöhtes Risiko für eine tuberkulöse Neuinfektion besteht [36]. THT oder IGRA (ggf. in Kombination) können laut CDC, kanadischen und britischen Empfehlungen eingesetzt werden [44]. Die European AIDS Clinical Society (EADS) empfiehlt (Oktober 2011) bei HIV-Erstdiagnose die Durchführung einer Röntgenthoraxuntersuchung, eines THTs (wenn die CD4-Zellzahl mehr als 400/Mikroliter beträgt) und eines IGRAs (in Gruppen mit besonders hohem Risiko) [45]. Bei Exposition sollte im Rahmen einer Umgebungsuntersuchung entsprechend den aktuellen DZK-Empfehlungen vorgegangen werden [5].

Zur Behandlung einer LTBI wird, wenn kein Verdacht auf eine Isoniazid-Resistenz vorliegt, bei HIV-positiven Menschen INH über neun Monate empfohlen [22].

Standard 17: Es sollte eine gründliche Evaluierung von Komorbiditäten vorgenommen werden, die das Ansprechen auf die antituberkulöse Behandlung oder den Therapieerfolg beeinflussen könnten. Beim Entwurf des Behandlungsplans sollte die behandelnde Institution zusätzliche Einrichtungen und Angebote zum Erreichen eines optimalen Behandlungsergebnisses bei jedem einzelnen Patienten 
ermitteln und diese in den individuellen Behandlungsplan integrieren. Dieser Plan sollte die Einschätzungen bezüglich weiterer Erkrankungen sowie Überweisungen für die Therapie dieser Erkrankungen beinhalten. Ein besonderes Augenmerk sollte auf Mitbehandlungen gelegt werden, die bekanntermaßen das Ergebnis der antituberkulösen Behandlung beeinflussen können. Dazu zählen beispielsweise die Behandlung von Diabetes mellitus, Drogenund Alkoholentzugs-/-entwöhnungsbehandlungen, Programme zur Tabakentwöhnung und andere psychosoziale Unterstützungsmöglichkeiten. Außerdem ist auf Überweisung zu Angeboten der Geburtsvorsorge oder der Säuglingspflege zu achten.

Kommentar:

Keine Ergänzung aus deutscher Sicht notwendig.

\section{Standards für die Öffentliche Gesundheit (Public Health)}

Standard 18: Alle an der Behandlung von Tuberkulosepatienten Beteiligten sollten sicherstellen, dass Personen, die engen Kontakt zu Patienten mit einer infektiösen Tuberkulose haben, internationalen Empfehlungen entsprechend untersucht und betreut werden. Die Prioritäten einer Umgebungsuntersuchung werden entsprechend der Wahrscheinlichkeit festgelegt, dass eine Kontaktperson 1) eine nicht-diagnostizierte Tuberkulose hat, 2) ein hohes Risiko aufweist, an aktiver Tuberkulose zu erkranken, wenn er/sie infiziert wurde, 3) gefährdet ist, an einer schweren Form von Tuberkulose zu erkranken, falls sich die Krankheit entwickelt, und 4) ein hohes Risiko aufweist, durch den Indexfall infiziert worden zu sein. Die höchste Priorität haben folgende Kontaktpersonen: Personen mit Symptomen, die eine Tuberkulose nahelegen, Kinder unter fünf Jahren, Kontaktpersonen mit bekannter Immunschwäche (oder Verdacht darauf), insbesondere HIV-Infektion, und Kontaktpersonen von Patienten mit MDR/XDR-Tuberkulose. Anderen engen Kontaktpersonen wird eine geringere Priorität gegeben.

\section{Kommentar:}

Bezüglich der deutschen Standards zur Durchführung von Umgebungsuntersuchungen sei auf die entsprechenden DZK-Empfehlungen verwiesen [5]. Die Priorisierung, die in Standard 18 dargestellt wird, muss in Ländern mit hoher TB-Prävalenz und geringen Ressourcen zwangsläufig erfolgen. Deutschland dagegen strebt letztendlich eine Eradikation der Tuberkulose an, sodass möglichst jegliche Übertragung der Erkrankung verhindert werden soll. Die Auswahl der Kontaktpersonen, die untersucht werden sollten, erfolgt nach zwei Kriterien: der Bewertung der Ansteckungsgefahr durch den Indexfall (maßgeblich vom bakteriologischen Befund und Symptomen/Verhalten (Husten) beeinflusst) und der Art, Intensität und Dauer des Kontakts zum Indexfall. Außerdem ist - wie in Standard 18 empfohlen - die Gefahr des Übergangs einer latenten Infektion mit Mycobacterium tuberculosis in eine aktive Erkrankung zu beachten. Einem erhöhten Erkrankungsrisiko sind dabei beispielsweise HIV-Infizierte, i.v.Drogenabhängige, Organtransplantierte, Personen mit chronischer Niereninsuffizienz oder Diabetes mellitus sowie Personen, die immunsupprimierende Medikamente (insbesondere TNF- $\alpha$ Inhibitoren [46]) einnehmen, ausgesetzt [5].

Für Personen, die mit Patienten mit ansteckungsfähiger MDR/ XDR-Tuberkulose Kontakt entsprechend den in [5] genannten Auswahlkriterien hatten, und bei welchen lege artis eine latente tuberkulöse Infektion nachgewiesen wurde, kann noch kein ausreichend evidenzbasiertes Vorgehen zur Chemoprävention empfohlen werden. Gegenwärtig wird für Deutschland bei erwachse- nen immunkompetenten Kontaktpersonen keine medikamentöse Prävention empfohlen, sondern stattdessen eine engmaschige Überwachung in der Regel mind. über 2 Jahre durch Experten gefordert [22]. Bei engen Kontaktpersonen mit LTBI und hohem Risiko im Weiteren zu erkranken (relevante Immunsuppression), ist eine Chemoprävention mit mindestens zwei Medikamenten in Abhängigkeit von dem Resistenzmuster des Erregers des Indexfalls zu erwägen (z.B. Fluorchinolon und EMB/Protionamid oder PZA und EMB über 12 Monate). Ein individuelles Vorgehen, Betreuung durch Experten und engmaschige Kontrollen aufgrund der häufigen Nebenwirkungen sollte dabei gesichert sein. Bei Kindern sollte Indikation und ggf. Zusammensetzung der Chemoprävention individuell von einem pädiatrischen Experten/Zentrum festgelegt werden.

Neueste Studien haben in den USA dazu geführt, dass von der CDC für bestimmte Personengruppen als Chemoprävention bei nachgewiesener LTBI nach Kontakt mit vollsensibler Tuberkulose Rifapentin (gewichtsadaptiert, siehe [47], max. 900 mg) in Kombination mit INH (15 mg/kg KG, max. $900 \mathrm{mg}$ ) einmal wöchentlich über drei Monate als überwachte Therapie (DOT) unter engem Monitoring möglicher Nebenwirkungen empfohlen wird [47]. Rifapentin ist in der EU zwar als „Orphan Drug“ zur Behandlung der Tuberkulose zugelassen, ist jedoch in Deutschland gegenwärtig noch nicht erhältlich, sodass dieses neue Therapieregime nicht Bestandteil aktueller Empfehlungen ist [22]. Auch bleiben weitere Studien abzuwarten, um zukünftige Empfehlungen entsprechend evidenzbasiert aussprechen zu können.

Standard 19: Kinder unter fünf Jahren und Personen jeden Alters mit einer HIV-Infektion, die enge Kontaktpersonen eines infektiösen Indexpatienten sind und die nach sorgfältiger Evaluation keine aktive Tuberkulose haben, sollten wegen zu vermutender latenter tuberkulöser Infektion mit Isoniazid behandelt werden.

\section{Kommentar:}

Die in Standard 19 genannten Gruppen weisen beide ein erhöhtes Erkrankungsrisiko nach Infektion auf. Daher wird empfohlen, nach Ausschluss einer Tuberkuloseerkrankung mit der prophylaktischen Gabe von Isoniazid zu beginnen, noch bevor eine latente tuberkulöse Infektion nachgewiesen werden kann (diagnostisches Fenster von ca. acht Wochen). In Deutschland sollte eine solche Chemoprophylaxe bei allen Personen mit Immunschwäche (unabhängig von deren Ursache) erwogen werden. Zur Gabe von Isoniazid stehen Alternativen zur Verfügung, die bei Unverträglichkeit oder nachgewiesener Isoniazid-Resistenz der Erreger des Indexfalls eingesetzt werden können [22]. Einen Sonderfall stellt hierbei die multiresistente Tuberkulose (MDRTB) dar (s.o.).

Bezüglich des Vorgehens im Anschluss an die Chemoprophylaxe, die auf acht bis zwölf Wochen begrenzt ist, bzw. generell bei immunkompetenten Kontaktpersonen über fünf Jahren sei auf die entsprechende Spezialliteratur verwiesen $[5,22]$.

Standard 20: Jede Gesundheitseinrichtung, die Patienten mit infektiöser Tuberkulose (oder den Verdacht darauf) versorgt, sollte einen angemessenen Plan für den Infektionsschutz bei Tuberkulose entwickeln und implementieren.

\section{Kommentar:}

Die Entwicklung eines Infektionspräventionskonzeptes wird für Krankenhäuser, Arztpraxen und andere Gesundheitseinrichtungen empfohlen bzw. ist gesetzlich vorgeschrieben (Arbeitsschutz, TRBA 250) $[30,48]$. Dieses Konzept ist regelmäßig dem aktuellen wissenschaftlichen Stand anzupassen. Eine Abstimmung mit 
dem hygienebeauftragten Arzt und dem Betriebsarzt sollte vorgenommen werden. Bezüglich der aktuellen Empfehlung zur Infektionsprävention inklusive konkreter Hygienemaßnahmen wird auf die entsprechende Fachliteratur verwiesen [30].

Standard 21: Alle an der Diagnostik und Behandlung von TB-Patienten Beteiligten müssen sowohl neue als auch trotz Vorbehandlung wiedererkrankte Fälle sowie die Behandlungsergebnisse dem zuständigen amtlichen Gesundheitsdienst gemäß den anzuwendenden Gesetzen melden.

Kommentar:

Die für Deutschland spezifische, gesetzlich geregelte Vorgehensweise wurde in den Kommentaren zu Standard 7 und 9 erläutert. Über die gesetzliche Meldepflicht hinaus sollte der Therapieverlauf in Abhängigkeit von den jeweiligen Umständen regelmäßig durch den ÖGD evaluiert werden. Eine Auskunftspflicht des behandelnden Arztes gegenüber dem Gesundheitsamt besteht gemäß §25 IfSG jedoch nicht.

\section{Danksagung}

Das DZK bedankt sich beim Bundesministerium für Gesundheit für die Unterstützung.

\section{Interessenkonflikt}

Die Autorinnen und Autoren geben an, dass keine Interessenkonflikte bestehen.

\section{Institute}

${ }^{1}$ Deutsches Zentralkomitee zur Bekämpfung der Tuberkulose, Berlin

${ }^{2}$ Lungenklinik Heckeshorn, HELIOS Klinikum Emil von Behring, Berlin

${ }^{3}$ Charité - Universitätsmedizin, Berlin

${ }^{4}$ Forschungszentrum Borstel

Zentrum für Pneumologie, Diakoniekrankenhaus Rotenburg (Wümme)

\section{Literatur}

1 Hopewell PC, Migliori GB, Raviglione MC. Tuberculosis care and control. Bull World Health Organ 2006; 84: 428

2 Migliori GB, Zellweger JP, Abubakar I et al. European Standards for Tuberculosis Care. Eur Respir J [im Druck]

3 Kardos R, Berck H, Fuchs K-H et al. Leitlinie der Deutschen Gesellschaft für Pneumologie und Beatmungsmedizin zur Diagnostik und Therapie von erwachsenen Patienten mit akutem und chronischem Husten. Pneumologie 2010; 64: 336-373

4 DEGAM-S3-Leitlinie Nr. 11: „Husten“ von. 2008: www.leitlinien.net [Zugriff am 29.1.2010]

5 Diel R, Loytved G, Nienhaus A et al. Deutsches Zentralkomitee zur Bekämpfung der Tuberkulose. Neue Empfehlungen für die Umgebungsuntersuchungen bei Tuberkulose. Pneumologie 2011; 65: 359-378 (kostenfrei über www.pneumologie.de/dzk/empfehlungen.html abrufbar)

6 Richter E, Beer J, Diel R et al. Tuberkulose Mykobakteriose, MiQ 5. München: Urban \& Fischer; 2010

7 Weltgesundheitsorganisation. Approaches to improve sputum smear microscopy for tuberculosis diagnosis. Expert group meeting report Geneva: WHO; 2009

8 Hillemann D, Rüsch-Gerdes S. Tuberkulose. Neuester Stand der modernen Mykobakteriendiagnostik. Pädiat Prax 2010; 75: 93-102

9 Hillemann D, Rüsch-Gerdes S, Richter E. Neue molekularbiologische Verfahren in der Tuberkulose-Diagnostik. Epidemiologisches Bulletin 2011; 34: $319-321$
10 Magdorf K, Bialek R, Detjen A et al. Tuberkulose und nicht tuberkulöse mykobakterielle Krankheiten. In: Deutsche Gesellschaft für Pädiatrische Infektiologie (DGPI), Hrsg. Infektionen bei Kindern und Jugendlichen. Stuttgart: Thieme; 5. Aufl. 2009

11 Robert Koch-Institut. Bericht zur Epidemiologie der Tuberkulose in Deutschland für 2009. Berlin: Robert Koch-Institut; 2011

12 Jin SJ, Kim JM, Kim HK et al. Histopathology and TB-PCR kit analysis in differentiating the diagnosis of intestinal tuberculosis and Crohn's disease. World J Gastroenterol 2010; 16: 2496-2503

13 Park DY, Kom JY, Choi KU et al. Comparison of polymerase chain reaction with histopathologic features for diagnosis of tuberculosis in formalin-fixed, paraffin-embedded histologic specimens. Arch Pathol Lab Med 2003; 127: 326 - 330

14 Pfyffer G. Stellenwert der Polymeraseketten-Reaktion (PCR) und anderer Amplifikationsverfahren in der Diagnostik der Tuberkulose. Pneumologie 2004; 58: 160 - 164

15 Lee H-M, Shin JW, Kim JY et al. HRCT and whole-blood Interferon-gamma Assay for the rapid diagnosis of smear-negative pulmonary tuberculosis. Respiration 2010; 79: 454-460

16 Übersicht der Änderungen zum EBM (Stand 1. Oktober 2011), die zum 1. Januar 2009 oder später in Kraft getreten sind. http://www.kbv.de/ 8429.html\#pkcvxdxijg [Zugriff am 18.11.2011]

17 Chung $C L$, Chen $C H$, Yeh $C Y$ et al. Early effective drainage in the treatment of loculated tuberculous pleurisy. Eur Respir J 2008; 31: 1261 1267

18 Von Groote-Bidlingmaier F, Bolliger CT. Der tuberkulöse Pleuraerguss Pneumologe 2010; 7: 343-348

19 Detjen A, Magdorf K. Besonderheiten der Kindertuberkulose. Pneumologie 2009; 63: $207-218$

20 Infektionsschutzgesetz. Bonn: Bundesgesetzblatt; Teil 1 G5702, 2000 1045

21 Forßßohm M, Loytved G, Königstein B, Hrsg. Handbuch Tuberkulose für Fachkräfte an Gesundheitsämtern. Düsseldorf: Akademie für öffentliches Gesundheitswesen; 2009

22 Schaberg T, Bauer T, Castell S et al. DZK, DGP. Empfehlungen zur Therapie, Chemoprävention und Chemoprophylaxe der Tuberkulose im Erwachsenen- und Kindesalter. Pneumologie 2012; 66, DOI 10.1055/s0031-1291619

23 Priwitzer M. Sicherung des Behandlungserfolges bei Tuberkulose. Entwurf einer Empfehlung für Deutschland durch den Arbeitskreis Tuberkulose im BVÖGD. 33. Informationsbericht des Deutschen Zentralkomitees zur Bekämpfung der Tuberkulose Berlin: DZK; 2010

24 http://www.g-ba.de/informationen/richtlinien/\#11/ [Zugriff am 25.11.2011]

25 http://www.sozialgesetzbuch-sgb.de/sgbxii/30.html [Zugriff am 24.11.2011]

26 Veen J, Raviglione $M$, Rieder $H L$ et al. Standardized tuberculosis treatment outcome monitoring in Europe. Eur Respir J 1998; 12: 505 - 510

27 Robert-Koch-Institut. Leitfaden zur Übermittlung von Fallberichten zur Tuberkulose. Berlin: Robert-Koch-Institut; 2004

28 World Health Organisation. Treatment of tuberculosis. Guidelines 4th ed 20104. WHO/HTM/TB/2009.420

29 Multidrug and extensively drug-resistant TB (M/XDR-TB). 2010 global report on surveillance and response $\mathrm{WHO} / \mathrm{HTM} / \mathrm{TB} / 2010.3$

30 Ziegler R, Just H-M, Castell S et al. Infektionsprävention bei Tuberkulose. Pneumologie 2012; 66, DOI 10.1055/s-0031-1291582 (kostenfrei über www.pneumologie.de/dzk/empfehlungen.html abrufbar)

31 Falzon D, Jaramillo E, Schünemann HJ et al. WHO guidelines for the programmatic management of drug-resistant tuberculosis: 2011 update. Eur Respir J 2011; 38: 516-528

32 Loddenkemper R, Hauer B. Resistente Tuberkulose. Große Herausforderung durch eine Weltepidemie. Dtsch Arztebl Int 2010; 107: 10 - 19

33 http://www.bundesaerztekammer.de/page.asp?his=1.100.1143 [Stand 2006]

34 Weimer T. Ärztliche Dokumentation: Im Streitfall auf der sicheren Seite. Dtsch Arztebl 2006; 103: [15]

35 Leitlinie der DAIG und ÖAG. Antiretrovirale Therapie der HIV-Infektion. Dtsch Med Wochenschr 2009; 134: S4 -S15

36 Kaplan JE, Benson C, Holmes KK et al. Guidelines for prevention and treatment of opportunistic infections in HIV-infected adults and adolescents: recommendations from CDC, the National Institutes of Health, and the HIV Medicine Association of the Infectious Diseases Society of America. MMWR Recomm Rep 2009; 58: 1 - 207

37 Török ME, Farrar JJ. When to start antiretroviral therapy in HIV-associated tuberculosis. N Engl J Med 2011; 365: 1538-1540 
38 Robert-Koch-Institut. Schätzung der Prävalenz und Inzidenz von HIVInfektionen in Deutschland. Epdemiologisches Bulletin 2011; 46: 415-425

39 Schieferstein C, Lange C. Tuberkulose. In: Hoffmann C, Rockstroh JK, Kamps BS, Hrsg. HIV.net 2008. Wuppertal-Beyenburg: Steinhäuser Verlag; 2008

40 Marais S, Pepper DJ, Marais BJ et al. HIV-associated tuberculous meningitis - diagnostic and therapeutic challenges. Tuberculosis 2010; 90: $367-374$

41 Meintjes G, Lawn S, Scano F et al. Tuberculosis-associated immune reconstitution inflammatory syndrome. Case definitions for use in resource-limited settings. Lancet Infect Dis 2008; 8: 516-523

42 Masur H, Kaplan JE, Holmes KK. Guidelines for preventing opportunistic infections among HIV-infected persons - 2002: Recommendations of the U. S. Public Health Service and the Infectious Diseases Society of America. Ann Intern Med 2002; 137: 435 - 477
43 Diel $R$, Loddenkemper $R$, Nienhaus A. Evidence based comparison of commercial interferon-gamma release assays for detecting active tuberculosis - a meta-analysis. Chest 2010; 137: 952 - 968

44 Horsburgh CR, Rubin EJ. Latent tuberculosis infection in the United States. N Engl J Med 2011; 364: 1441 - 1448

45 European AIDS Clinical Society (EACS). Guidelines, Version 6.0. Paris: EACS; 2011: http://www.europeanaidsclinicalsociety.org/

46 Diel R, Hauer B, Loddenkemper R et al. Empfehlungen für das Tuberkulosescreening vor Gabe von TNF-alpha-Inhibitoren bei rheumatischen Erkrankungen. Pneumologie 2009; 63: 329-334

47 Jereb J, Goldberg SV, Powell $K$ et al. Recommendations for use of an isoniazid-rifapentine regimen with direct observation to treat latent Mycobacterium tuberculosis infection. MMWR 2011; 60: 1650 - 1652

48 TRBA-250. http://www.baua.de/nn_15116/de/Themen-von-A-Z/Biologische-Arbeitsstoffe/TRBA/pdf/TRBA-250.pdf[Zugriffam25.10.2011] 